\title{
Persistence Length Changes Dramatically as RNA Folds
}

\author{
G. Caliskan ${ }^{1,2}$, C. Hyeon ${ }^{3}$, U. Perez-Salas ${ }^{4,5}$, R. M. Briber ${ }^{6}$, S. A. Woodson ${ }^{7}$, and D. Thirumalai ${ }^{3}$ \\ ${ }^{1}$ T. C. Jenkins Department of Biophysics, Johns Hopkins University, 3400 N. Charles St., Baltimore, MD 21218 \\ ${ }^{2}$ Center for Neutron Research, National Institute of Standards and Technology, Gaithersburg, MD 20899 \\ ${ }^{3}$ Biophysics Program, Institute For Physical Science and Technology, \\ University of Maryland, College Park, MD 20742 \\ ${ }^{4}$ Center for Neutron Research, National Institute of Standards and Technology, Gaithersburg, MD 20899 \\ ${ }^{5}$ Department of Physiology and Biophysics, University of California at Irvine, Irvine, CA 92697 \\ ${ }^{6}$ Department of Materials Science and Engineering, \\ University of Maryland, College Park, MD 20742 \\ ${ }^{7}$ T. C. Jenkins Department of Biophysics, Johns Hopkins University, 3400 N. Charles St., Baltimore, MD 21218
}

(Dated: October 30, 2018)

\begin{abstract}
We determine the persistence length, $l_{p}$, for a bacterial group I ribozyme as a function of concentration of monovalent and divalent cations by fitting the distance distribution functions $P(r)$ obtained from small angle X-ray scattering intensity data to the asymptotic form of the calculated $P_{W L C}(r)$ for a worm-like chain (WLC). The $l_{p}$ values change dramatically over a narrow range of $\mathrm{Mg}^{2+}$ concentration from $\sim 21 \AA$ in the unfolded state $(\mathbf{U})$ to $\sim 10 \AA$ in the compact $\left(\mathrm{I}_{\mathrm{C}}\right)$ and native states. Variations in $l_{p}$ with increasing $\mathrm{Na}^{+}$concentration are more gradual. In accord with the predictions of polyelectrolyte theory we find $l_{p} \propto 1 / \kappa^{2}$ where $\kappa$ is the inverse Debye-screening length.
\end{abstract}

Elucidating the mechanisms by which RNA molecules self-assemble to form three dimensional structures is a challenging problem [1, 2, 3, 4]. Because the native state $(\mathbf{N})$ cannot form without significantly neutralizing the negative charge on [5, 6] the phosphate group, RNA folding is sensitive to the valence, size, and shape of the counterions. At low counterion concentrations $(C)$ RNA is unfolded $(\mathbf{U})$ in the sense that it contains isolated stretches of base-paired stem loops that have large dynamical fluctuations. When $C>C_{m}$, the midpoint of the transition from $\mathbf{U}$ to the $\mathbf{N}$, RNA becomes compact as a result of formation of tertiary contacts. For many RNA molecules, such as the Tetrahymena ribozyme and RNase $\mathrm{P}$, folding to the native state is preceded by the formation of multiple metastable kinetic intermediates (I) [1, 2, 7].

The large dynamic conformational fluctuations in the $\mathbf{U}$ and $\mathbf{I}$ states make it difficult to characterize their structures. However, small angle scattering experiments can be used to determine the shape of RNA as it folds. The conformation of RNA in the $\mathbf{U}, \mathbf{N}$, and the $\mathbf{I}$ states is characterized by $R_{g}$, the radius of gyration, and $l_{p}$, the persistence length. Small Angle X-ray Scattering (SAXS) 2, 8, 9, 10, 11] and Small Angle Neutron Scattering (SANS) 12] experiments have been used to obtain $R_{g}$ as a function of counterions for a number of RNA molecules. In contrast, $l_{p}$, which is a function of $C$ and valence and shape of counterions, is more difficult to obtain.

In this letter, we use SAXS data and theoretical results for the worm-like chain (WLC) to obtain $l_{p}$ for a 195 nucleotide group I ribozyme from pre-tRNA(Ile) of the Azoarcus bacterium as a function of $C$ for monova- lent and divalent counterions. The major conclusions of the present study are: (i) The experimentally determined distance distribution functions $P(r)$ can be accurately fit using the theoretical results for wormlike chains for $r / R_{g}>1$ where $R_{g}$ is the radius of gyration of RNA. The $l_{p}$ values, which were calculated by fitting $P(r)$ to $P_{W L C}(r)$ for $r>R_{g}$, change dramatically from $l_{p} \simeq 21 \AA$ in the $\mathbf{U}$ state to $l_{p} \simeq 10$ $\AA$ in the compact conformation. (ii) The large reduction in $l_{p}$ occurs abruptly over a narrow concentration range in $\mathrm{Mg}^{2+}$ whereas the decrease of $l_{p}$ in $\mathrm{Na}^{+}$is gradual. This result suggests that the compaction of RNA resembles a first order transition in the presence of multivalent counterions. (iii) For both $\mathrm{Na}^{+}$and $\mathrm{Mg}^{2+}$, the persistence length scales as $l_{p} \simeq l_{D}^{2}$ where $l_{D}$ is the Debye-screening length. From this finding, which is in accord with the predictions of polyelectrolyte theory, we find that the intrinsic persistence length of RNA is $l_{p}^{o} \simeq 10 \AA$.

The Azoarcus ribozyme was transcribed in vitro as described previously [13]. We carried out SAXS measurements at Argonne National Lab Advanced Photon Source (BIOCAT) beamline using $1.05 \AA \mathrm{X}$-rays that corresponds to $11.8 \mathrm{keV}$ in energy. A sample to detector distance of $1.89 \mathrm{~m}$ allowed us to probe momentum transfer $(Q)$ in the range from $(\sim 0.007$ to $\sim 0.266) \AA^{-1}$. A quartz capillary flow cell was used to minimize the radiation damage due to X-ray exposure of a given RNA chain [9]. The measurements at various flow rates showed that $\mathrm{X}$-ray radiation damage is negligible. Each measurement was averaged from four separate exposures of two seconds each. The SAXS profiles were corrected for the background signal which was 

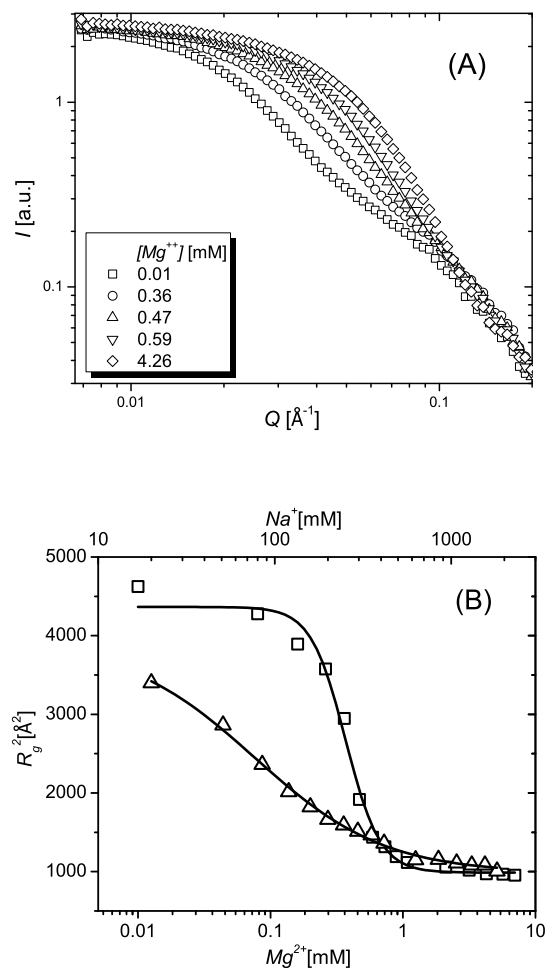

FIG. 1: (A) Scattering intensity $I(Q)$ as a function of $Q$ for 195 nucleotide Azoarcus ribozyme at different as a function of $\mathrm{Mg}^{2+}$ concentration in $20 \mathrm{mM}$ Tris buffer, $\mathrm{pH}=7.5$ at $32^{\circ} \mathrm{C}$. The values of $\mathrm{Mg}^{2+}$ concentrations are given in the inset. (B) The dependance of $R_{g}^{2}$ on $\mathrm{Mg}^{2+}$ (squares) and $\mathrm{Na}^{+}$(triangles) concentration. Solid line is the fit using Hill equation in the form $A\left(1-\left[M g^{2+}\right]^{n} /\left(C_{m}^{n}+\left[M g^{2+}\right]^{n}\right)\right)+$ $y_{o}$, where $A, n$ and $y_{o}$ are adjustable parameters. We find $n$ is 3.33 and 1.20 for $\mathrm{Mg}^{2+}$ and $\mathrm{Na}^{+}$, respectively.

measured at same buffer concentrations in the absence of RNA. Using the background corrected SAXS intensity as a function of $Q,(I(Q))$ (Fig. (1A)) the distance distribution function, $P(r)$, was calculated by an indirect inversion algorithm [14]. The square of the radius of gyration is given by $R_{g}^{2}=\int r^{2} P(r) \mathrm{d} r / \int 2 P(r) \mathrm{d} r$.

Single molecule measurements of RNA subject to tension and our analysis of PDB structure have shown that the force-extension curves can be fit using a wormlike chain (WLC) model [15]. Based on these studies we assume that RNA is a WLC for which $P(r)$ cannot be calculated analytically. However, a simple theoretical expression has been derived [16] for the end-to-end $R_{E}$ distribution using a mean-field model of WLC. We expect that asymptotically $\left(r>>R_{g}\right)$ the behavior of $P(r)$ and the distribution of $R_{g}$ or $R_{E}$ should have the same functional form. Thus, for large $r$ we predict
$P(r)$ should decay as $[6]$

$$
P_{W L C}(r) \sim \exp \left(-\frac{1}{1-x^{2}}\right)
$$

where $x=l_{p} r / R_{g}^{2}$.

At all concentrations of $\mathrm{Na}^{+}$and $\mathrm{Mg}^{2+}$, Eq. (3) fits the data extremely accurately as long as $r / R_{g}>1$ (Fig. (2)). The excellent fits in Fig. (2) allows us to determine $l_{p}$ as a function of the counterion concentration. When RNA is unfolded at low $\mathrm{Na}^{+}$or $\mathrm{Mg}^{2+}$ concentration, $l_{p} \simeq 21 \AA$ with $R_{g} \simeq 65 \AA$. As the concentration of $\mathrm{Na}^{+}$increases from about $(20$ - 200) $\mathrm{mM}, l_{p}$ gradually decreases. There is a sharp decrease in $R_{g}$ when $\left[\mathrm{Na}^{+}\right] \simeq 250 \mathrm{mM}$ that is accompanied by a large reduction in the persistence length to $l_{p} \simeq 10 \AA$. The changes in $l_{p}$ are even more dramatic in $\mathrm{Mg}^{2+}$ (Fig. (2B)). As $\mathrm{Mg}^{2+}$ increases from $0.01 \mathrm{mM}$ to $0.26 \mathrm{mM}$ the persistence length changes only by about $3 \AA$ from $l_{p} \simeq 21 \AA \quad(0.01 \mathrm{mM})$ to $l_{p} \simeq 18.3 \AA(0.26 \mathrm{mM})$. In this concentration range $R_{g}$ decreases from $65 \AA$ to $60 \AA$. A further increase in $\mathrm{Mg}^{2+}$ to $4.26 \mathrm{mM}$ leads to a reduction in $R_{g}$ to about $31 \AA$ with a dramatic decrease in $l_{p}$ to about $10 \AA$. The near discontinuous change in $R_{g}$ in $\mathrm{Mg}^{2+}$ (Fig. (1B)) suggests a first-order coil-globule transition in $\mathrm{Mg}^{2+}$. While less common in neutral homopolymers, a discontinuous coil-globule transition has been predicted to occur in strongly charged polyelectrolytes [17].

To complement the experimental studies we calculated the $P(r)$ functions for the native three dimensional structure of Azoarcus ribozyme using the coordinates from X-ray crystallography crystal structure [18] (PDB id: 1U6B) and a model based on sequence comparison [13. The computations were done using the coordinates of only the heavy atoms $(\mathrm{C}, \mathrm{O}, \mathrm{P}$, and $\mathrm{N})$. To compare the results obtained from crystal structures and SAXS data, we used only the heavy atom coordinates for chain B (excluding nucleotides 1 and 197) from 1U6B structure to compute $P(r)$. Similarly, the exon fragments were excluded from the Westhof model.

The $P(r)$ function from the SAXS data for the $\mathbf{N}$ state and those obtained using the X-ray structure and the Westhof model are in good agreement with each other and the SAXS data(Fig. 3A). The radii of gyration for the native state calculated using $\left(R_{g}^{N}\right)^{2}=\frac{1}{2 N^{2}} \sum_{i} \sum_{j}\left(r_{i}-r_{j}\right)^{2}$ for the X-ray structure and the Westhof model are $31.1 \AA$ and $30.7 \AA$ respectively. These values agree well with the results from the SAXS data $\left(R_{g}^{N}=30.9 \AA\right)$. The $l_{p}$ for the native state obtained by fitting the crystal structure $P(r)$ to Eq. (3) is $11 \AA$, while for the Westhof model we obtain $l_{p} \approx 10.8 \AA$. The good agreement between the crystal 


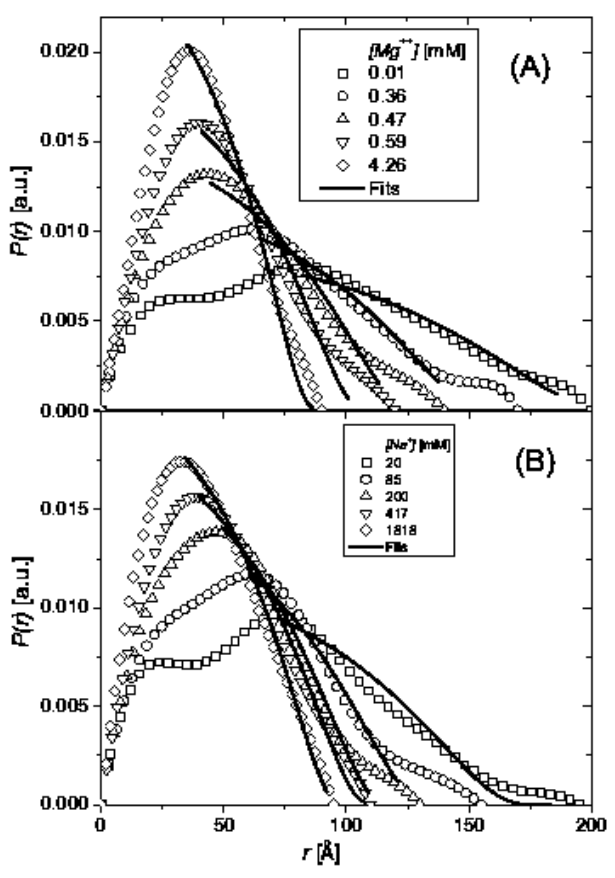

FIG. 2: Distance distribution functions. (A) $P(r)$ functions at various $\mathrm{Mg}^{2+}$ concentrations at $32^{\circ} \mathrm{C}$ are obtained using Eq. (1) with $Q_{\min }=0.07 \AA$ and $Q_{\max }=0.1 \AA$. The solid lines are fits of $P(r)$ to Eq.(3) (B) Same as (A) except the counterion is $\mathrm{Na}^{+}$. The concentrations of counterions are given in the insets.

structure (or the Westhof model) and the SAXS measurement for $P(r)$ and $l_{p}$ in the $\mathrm{I}_{\mathrm{C}}$ state suggests that the effects of complexation and interparticle interactions in the SAXS experiments are negligible.

Since RNA appears to be a charged worm-like polyelectrolyte, it is of interest to ascertain if the dependance of $l_{p}$ on the Debye-screening length conforms to the theoretical predictions [19, 20]. The dependence of $l_{p}$ on the square of the Debye-screening length $\left(l_{D}^{-2}=\kappa^{2}=8 \pi l_{B} I\right.$ where $l_{B}=e^{2} / 4 \pi \epsilon k_{B} T$ is the Bjerrum length and $I$ is the ionic strength) is linear for both $\mathrm{Na}^{+}$and $\mathrm{Mg}^{2+}$ (Fig. (3B)). For both flexible [21, 22] and stiff polyelectrolytes [19, 20] it has been shown that $l_{p}=l_{p}^{o}+l_{p}^{e l}$ where $l_{p}^{o}$ is the intrinsic persistence length and the electrostatic contribution is $l_{p}^{e l} \propto 1 / \kappa^{2}$. Deviation from the OSF predictions can occur for finite-sized flexible polyelectrolytes. However, we do not expect such deviations because RNA is intrinsically stiff. Surprisingly, over the range of $\mathrm{Na}^{+}$and $\mathrm{Mg}^{2+}$ concentrations in which the Azoarcus ribozyme undergoes the $\mathbf{U} \rightarrow \mathrm{I}_{\mathrm{C}}$ transition, the experimental data confirm the predictions of polyelectrolyte theory. From the linear fits of $l_{p}$ to $\kappa^{-2}$ (Fig. (3B)) we obtain $l_{p}^{o} \simeq 10 \AA$ which is similar to those found
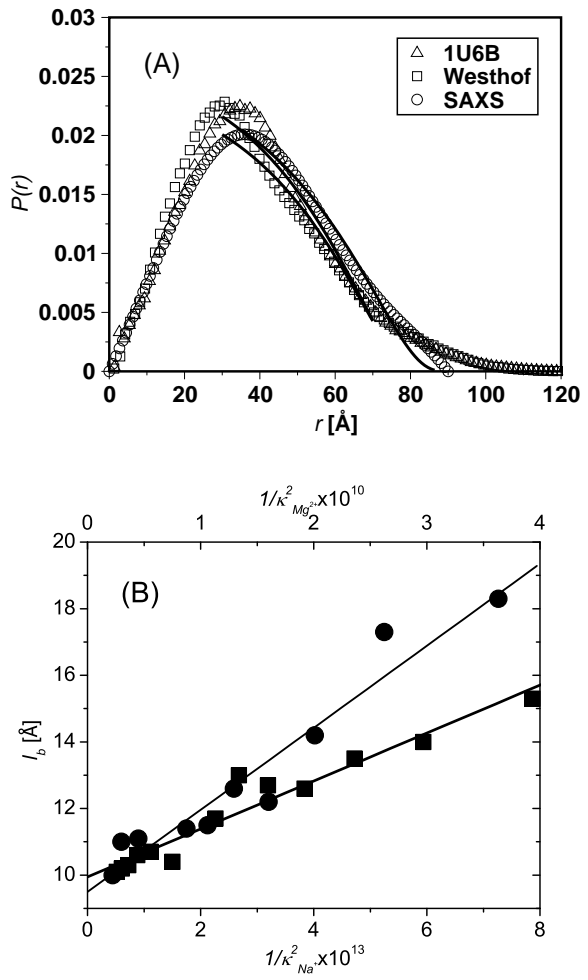

FIG. 3: (A) Calculation of $P(r)$ using the coordinates of the heavy atoms from the chain $\mathrm{B}$ of the crystal structure (1U6B), the Westhof model [13], and SAXS data. The symbols are given in the inset. The solid lines show the fits using $P_{W L C}(r)$ in the range $30 \AA$ to $70 \AA$. In this range the root mean square deviation of the $P(r)$ for the $1 \mathrm{U} 6 \mathrm{~B}$ from $P_{W L C}(r)$ is $0.13 \AA^{-1}$ and for the Westhof model 13] it is $0.10 \AA^{-1}$. The correlation coefficient of the fits of $P(r)$ to $P_{W L C}(r)$ is 0.98 . (B) Dependence of $l_{p}$ on $1 /$ $\kappa^{2}$ in $\mathrm{Mg}^{2+}$ (solid circles) and in $\mathrm{Na}^{+}$(solid squares). Lines represent fits to the data. Note that the $1 / \kappa^{2}$ scale for $\mathrm{Mg}^{2+}$ is given on top.

for single stranded DNA [23, 24].

To assess if $P(r)$ for WLC can be used to fit scattering measurements on other RNA molecules we used Eq. (3) and SAXS data for RNase P [9] as a function of $\mathrm{Mg}^{2+}$ concentration. Unlike the Azoarcus ribozyme, folding of RNase $\mathrm{P}$ is best described using three states, namely, $\mathbf{U}$, an intermediate $\mathbf{I}$, and the native state, $\mathbf{N}$ [9]. The $\mathbf{I}$ state is populated in the $\mathrm{Mg}^{2+}$ range $0.02<$ $\mathrm{Mg}^{2+}<0.2$. From the accurate fit of the SAXS data using Eq. (3) for $r / R_{g}>1$, the $l_{p}$ values are found to be $24.5 \AA, 14.1 \AA$, and $11.6 \AA$ in the $\mathbf{U}, \mathbf{I}$, and $\mathbf{N}$ states respectively. The largest decrease in $l_{p}$ and the associated $R_{g}$ occurs in the $\mathbf{U} \rightleftharpoons \mathbf{I}$ transition, which is consistent with the notion that the early event in RNA collapse is initiated by counterion condensation [3]. 


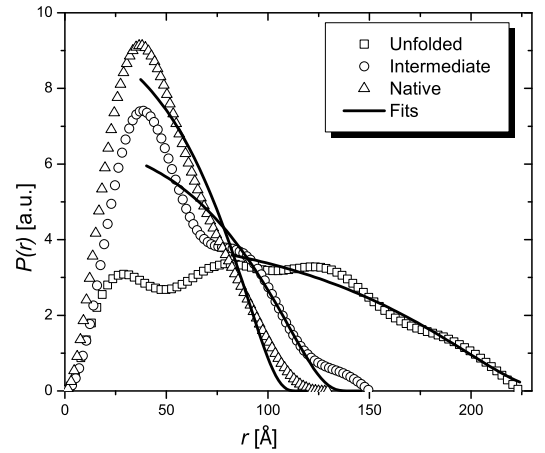

FIG. 4: $P(r)$ for RNase $\mathrm{P}$ from reference [9]. The distance distribution function was calculated from $I(q)$ using Eq. (1) with a different numerical Fourier Transform method 25]. The $\mathbf{U}, \mathbf{I}$ and $\mathbf{N}$ states are for $\mathrm{Mg}^{2+}$ concentrations $0,0.1$, and $10 \mathrm{mM}$ respectively. The lines are fits using Eq. (3).

The present work shows that the size and flexibility of RNA molecules as a function of counterion concentration can be obtained using scattering experiments and the WLC model. Given that RNA is a highly branched and charged polymer, it is surprising that the distance distribution functions can be described using elasticity-based polymer models for $r / R_{g}>1$. Although the structural basis for such a behavior is not obvious, the demonstration that single stranded DNA 24], double stranded DNA [26], and polypeptide chains [27] also behave like WLC suggests that for compatible interactions between biomolecules the local flexibility should be similar.

We are grateful to T. R. Sosnick for providing the $P(r)$ data for RNase $\mathrm{P}$ in tabular form. This work was supported in part by a grant from the National Science Foundation to DT (grant number 05-14056) and the National Institutes of Health to SAW.

[1] D. K. Treiber and J. R. Williamson, Curr. Opin. Struct. Biol. 9, 339 (1999).

[2] T. R. Sosnick and T. Pan, Curr. Opin. Struct. Biol. 13, 309 (2003).

[3] D. Thirumalai and C. Hyeon, Biochemistry 44, 4957 (2005).

[4] P. Brion and E. Westhof, Ann. Rev. Biophys. Biomol. Struct. 26, 113 (1997).

[5] V. K. Misra and D. E. Draper, Biopolymers 48, 113
(1998).

[6] D. Thirumalai, N. Lee, S. A. Woodson, and D. K. Klimov, Ann. Rev. Phys. Chem. 52, 751 (2001).

[7] J. Pan, D. Thirumalai, and S. A. Woodson, J. Mol. Biol. 273, 7 (1997).

[8] K. Takamoto, R. Das, Q. He, S. Doniach, M. Brenowitz, D. Herschlag, and M. R. Chance, J. Mol. Biol. 343, 1195 (2004).

[9] X. Fang, K. Littrell, X. J. Yang, S. J. Henderson, S. Siefert, P. Thiyagarajan, T. Pan, and T. R. Sosnick, Biochemistry 39, 11107 (2000).

[10] R. Russell, I. S. Millett, S. Doniach, and D. Herschlag, Nat. Struct. Biol. 7, 367 (2000).

[11] R. Russell, I. S. Millettt, M. W. Tate, L. W. Kwok, B. Nakatani, S. M. Gruner, S. G. J. Mochrie, V. Pande, S. Doniach, D. Herschlag, et al., Proc. Natl. Acad. Sci. USA 99, 4266 (2002).

[12] U. A. Perez-Salas, P. Rangan, S. Krueger, R. M. Briber, D. Thirumalai, and S. A. Woodson, Biochemistry 43, 1746 (2004).

[13] P. Rangan, B. Masquida, E. Westhof, and S. A. Woodson, Proc. Natl. Acad. Sci. U.S.A. 100, 1574 (2003).

[14] A. V. Semenyuk and D. I. Svergun, http://www.emblhamburg.de/ExternalInfo/Research/Sax/gnom.html (2005).

[15] J. Liphardt, B. Onoa, S. B. Smith, I. Tinoco, and C. Bustamante, Science 292, 733 (2001).

[16] D. Thirumalai and B.-Y. Ha, Theoretical and Mathematical Models in Polymer Research (Academic Press, San Diego, 1998), chap. Statistical Mechanics of Semiflexible Chains: A Mean Field Variational Approach, pp. $1-35$.

[17] B. Y. Ha and D. Thirumalai, Phys. Rev. A 46, R3012 (1992).

[18] P. L. Adams, M. R. Stahley, A. B. Kosek, J. M. Wang, and S. A. Strobel, Nature 430, 45 (2004).

[19] T. Odijk, J. Polym.Sci. B 15, 477 (1977).

[20] J. Skolnick and M. Fixman, Macromolecules 10, 944 (1977).

[21] B. Y. Ha and D. Thirumalai, J. Chem. Phys. 110, 7533 (1999)

[22] R. R. Netz and H. Orland, Euro. Phys. J. B 8, 81 (1999), M. Manghi and R. R. Netz, Euro. Phys. J. E 14, 67 (2004).

[23] C. Rivetti, C. Walker, and C. Bustamante, J. Mol. Biol. 280 (1998).

[24] B. Tinland, A. Pluen, J. Sturm, and G. Weill, Macromolecules 30, 5763 (1997).

[25] D. I. Svergun and A. V. Semenyuk, http://www.srs.dl.ac.uk/ncd/computing/manual.gnom.html (2000).

[26] S. B. Smith, L. Finzi, and C. Bustamante, Science 258, 1122 (1992).

[27] B. Schuler, E. A. Lipman, P. J. Steinbach, M. Kumke, and W. A. Eaton, Proc. Natl. Acad. Sci. USA 102, 2754 (2005). 